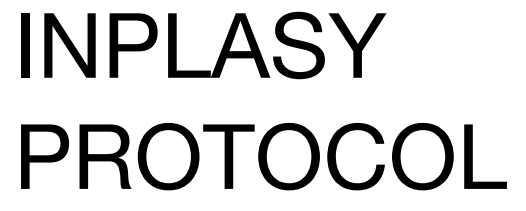

To cite: Zhang et al.

Associations between statin use and osteoarthritis risk and progression: a systematic review and meta-analysis. Inplasy protocol 202040160. doi:

10.37766/inplasy2020.4.0160

Received: 23 April 2020

Published: 23 April 2020

Corresponding author:

Xueyong Liu

liuxysjh@sj-hospital.org

Author Affiliation:

Shengjing Hospital of China

Medical University

Support: None.

Review Stage at time of this submission: Risk of bias assessment.

Conflicts of interest: No specific funding was received from any funding bodies in the public, commercial or not-forprofit sectors to carry out the work described in this manuscript.

\section{Associations between statin use and osteoarthritis risk and progression: a systematic review and meta-analysis}

\author{
Zhang, Z1; Deng, CB2; Ma, X33 Liu, XY4.
}

Review question / Objective: Populations: Included participants were over 18 years of age, had a prescription or a medication of statins. Non-users were in the same population as statin users and with no any statin medication. Interventions: Use of statin (regardless of kind, duration, and dosage), may be in any baseline condition, any co-medication, or any time-history of use. Comparison: Osteoarthritis risk, progression and/or other available indexes like operations due to osteoarthritis, conditions of statin use (duration, dosage) were also estimated if data were available. Outcomes: OR/HR/ RRs concerning OA incidence, progression and/or others obtained from included subjects that were expected.

Condition being studied: Osteoarthritis (OA) is the most common form of degenerative joint disease, and the major cause of pain and disability in the elderly. In addition to a low quality of life, OA imposes a huge social and economic burden. The etiopathogenesis of $O A$ is currently believed to be multifactorial, involving ageing, mechanical loading, metabolism and inflammation. Metabolic factors, such as dyslipidemia and hypercholesterolemia, have also been proved to be significantly associated with $O A$. The proinflammatory effects of lipids, adipokine-linked proinflammatory cytokines and pathways, and atherosclerotic vascular disease, have been reported associated with OA pathogenesis. There is now a growing interest in the use of statins as potential disease-modifying agents for $O A$. However, in previous reported clinical studies, statin use may be linked to adverse effects in musculoskeletal system and metabolisms, but it remains controversial. Meanwhile, it is inconclusive whether statin use is related to OA development and progression. Therefore, the purpose of this study was to conduct a systematic review and meta-analysis to estimate the effect of statin use on OA risk and progression.

INPLASY registration number: This protocol was registered with the International Platform of Registered Systematic Review and Meta-Analysis Protocols (INPLASY) on 23 April 2020 and was last updated on 23 April 2020 (registration number INPLASY202040160).

\section{INTRODUCTION}

Review question / Objective: Populations: Included participants were over 18 years of age, had a prescription or a medication of statins. Non-users were in the same population as statin users and with no any statin medication. Interventions: Use of 
statin (regardless of kind, duration, and dosage), may be in any baseline condition, any co-medication, or any time-history of use. Comparison: Osteoarthritis risk, progression and/or other available indexes like operations due to osteoarthritis, conditions of statin use (duration, dosage) were also estimated if data were available. Outcomes: OR/HR/RRs concerning OA incidence, progression and/or others obtained from included subjects that were expected.

Condition being studied: Osteoarthritis (OA) is the most common form of degenerative joint disease, and the major cause of pain and disability in the elderly. In addition to a low quality of life, OA imposes a huge social and economic burden. The etiopathogenesis of $O A$ is currently believed to be multifactorial, involving ageing, mechanical loading, metabolism and inflammation. Metabolic factors, such as dyslipidemia and hypercholesterolemia, have also been proved to be significantly associated with OA. The proinflammatory effects of lipids, adipokine-linked proinflammatory cytokines and pathways, and atherosclerotic vascular disease, have been reported associated with $O A$ pathogenesis. There is now a growing interest in the use of statins as potential disease-modifying agents for OA. However, in previous reported clinical studies, statin use may be linked to adverse effects in musculoskeletal system and metabolisms, but it remains controversial. Meanwhile, it is inconclusive whether statin use is related to OA development and progression. Therefore, the purpose of this study was to conduct a systematic review and metaanalysis to estimate the effect of statin use on OA risk and progression.

\section{METHODS}

Participant or population: Included participants were over 18 years of age, had a prescription or a medication of statins.

Intervention: Use of statin (regardless of kind, duration, and dosage), may be in any baseline condition, any co-medication, or any time-history of use.
Comparator: Non-users of statin were in the same population as statin users and with no any statin medication.

Study designs to be included: Randomized controlled trials, prospective or retrospective cohort studies, case-control and cross-sectional studies were included.

Eligibility criteria: Studies were selected based on the following criteria: (1) data of relevant studies concerning statin use and OA risk and/or progression, including baseline data of other related studies; (2) studies with available data or conclusion (systematic reviews only).

Information sources: An online systematic search was conducted using the electronic databases of MEDLINE (PubMed), Cochrane library, Embase, Web of Science and Scopus, from the date of database inception until January 1, 2020. Search strategy: ("osteoarthritis"[MeSH Terms] OR "osteoarthritis" [AII Fields] OR "degenerative arthritis"[All Fields]) AND ("hydroxymethylglutaryl-coa reductase inhibitors"[Pharmacological Action] OR "hydroxymethylglutaryl-coa reductase inhibitors"[MeSH Terms] OR statin[MeSH Terms] OR "atorvastatin"[All Fields] OR "cerivastatin" [AII Fields] OR "crilvastatin"[All Fields] OR "Lovastatin"[All Fields] OR "mevastatin"[All Fields] OR "pitavastatin"[AII Fields] OR "Pravastatin"[AII Fields] OR "Rosuvastatin"[AII Fields] OR "Simvastatin"[All Fields]). No language limitation was set. After the process of primary retrieval and study selection, the references of relevant studies and grey literaturewere also searched to avoid omission.

Main outcome(s): OR/HR/RRs of OA incidence or progression of the statin users versus non-users of population studied.

Additional outcome(s): Trend tests of duration/dosage-related risk.

Quality assessment / Risk of bias analysis: Cross-sectional, case-control and cohort studies are assessed using the Newcastle- 
Ottawa Scale (NOS) developed for these studies, and randomized controlled trials (RCTs) are assessed with the Cochrane risk-of-bias criteria.

Strategy of data synthesis: For the eligible data, meta-analysis will be performed using the $R$ package meta software (version 3.6.3). Detailed participants' data and OR/ HR/RRs of statin users vs non-users will be used. Pooled data have low heterogeneity if $P$ is greater than 0.1 and I-square was less than $50 \%$. In these cases, a fixed effects model will be used; otherwise, a random effects model will be used. Statistical analyses are two-sided and $a$ P value less than 0.05 is considered significant. The Zscore will be evaluated by the $P$ value of the two-sided U-test for effect estimation. For the data deem ineligible for metaanalysis, we will list and analyse all the involved data without statistical synthesis.

Subgroup analysis: Subgroup analysis will be conducted based on demographics, clinical characteristics, or adjusted confounders.

Sensibility analysis: A sensitivity analysis will be performed to investigate sources of heterogeneity and determine if excluding any individual study alters our results.

Language: No language limit was set.

Country(ies) involved: China.

Keywords: Statin, osteoarthritis, metaanalysis, risk, progression.

\section{Contributions of each author:}

Author 1 - Zhan Zhang - Author 1 performed project design, study selection, data extraction and assessment, statistical analysis, and manuscript writing.

Author 2 - Chunbo Deng - Author 2 performed study selection, data extraction, data analysis.

Author 3 - Xun Ma - Author 3 performed study selection, data extraction and data analysis.

Author 4 - Xueyong Liu - Author 4 was corresponding author, read, provided feedback and approved the manuscript.
Search strategy: Pubmed (osteoarthriti[All Fields] OR osteoarthritic[All Fields] OR osteoarthritic'[AII Fields] OR osteoarthritics[AII Fields] OR osteoarthritides[AII Fields] OR osteoarthrities [AII Fields] OR osteoarthritis [AII Fields] OR osteoarthritis '[AII Fields] OR osteoarthritisand[AII Fields] OR osteoarthritisassociated[All Fields] OR osteoarthritisderived[All Fields] OR osteoarthritisis [AII Fields] OR osteoarthritislike[AII Fields] OR osteoarthritisprogression[AllFields] OR osteoarthritisrabbit[AII Fields] OR osteoarthritiss [AII Fields] OR osteoarthritistis[AII Fields] OR osteoarthrititis [AII Fields] OR osteoarthritits [AII Fields]) OR (("osteoarthritis"[MeSH Terms] OR "osteoarthritis"[AII Fields]) OR (osteoarthritc [AII Fields] OR osteoarthrite[AII Fields] OR osteoarthrites [AII Fields] OR osteoarthrith [AII Fields] OR osteoarthrithis [AII Fields] OR osteoarthriti[AII Fields] OR osteoarthritic[AII Fields] OR osteoarthritic'[AII Fields] OR osteoarthritics [AII Fields] ORosteoarthritides[AII Fields] OR osteoarthrities [AII Fields] OR osteoarthritis [AII Fields] OR osteoarthritis '[AII Fields] OR osteoarthritisand [AII Fields] OR osteoarthritisassociated[All Fields] OR osteoarthritisderived[AII Fields] OR osteoarthritisis [AII Fields] OR osteoarthritislike[AII Fields] OR osteoarthritisprogression[All Fields] OR osteoarthritisrabbit[AII Fields] OR osteoarthritiss [AII Fields] OR osteoarthritistis [AII Fields] OR osteoarthrititis [AII Fields] OR osteoarthritits [AII Fields] OR osteoarthrits[AII Fields]) OR (osteo arthrite[All Fields] OR osteo arthrites[AII Fields] OR osteo arthritic[All Fields] OR osteo arthritics[AII Fields] OR osteo arthritis[All Fields]) OR (osteoarthro[All Fields] OR osteoarthroapthy[All Fields] OR osteoarthrocutaneous[AII Fields] OR osteoarthrodermopathic[All Fields] OR osteoarthrodesis[AII Fields] OR 
osteoarthrodysplasia[AII Fields] OR osteoarthrography[AII Fields] OR osteoarthrologiai[AII Fields] OR osteoarthrological[AII Fields] OR osteoarthrolog y [AII Fields ] ORosteoarthromuscular[All Fields] OR osteoarthromusculovascular[All Fields] OR osteoarthromyalgias [All Fields] OR osteoarthromyopathies[All Fields] OR osteoarthroonychodysplasie[All Fields] OR osteoarthropathe [AII Fields] OR osteoarthropathi[AII Fields] OR osteoarthropathi'aja[AII Fields] OR osteoarthropathia[AII Fields] OR osteoarthropathiaja[AII Fields] OR osteoarthropathic [All Fields] OR osteoarthropathie[AII Fields] OR osteoarthropathien[All Fields] OR osteoarthropathies[AII Fields] OR osteoarthropathique[AII Fields] OR osteoarthropathy [AII Fields] OR osteoarthropathy1[AII Fields] OR osteoarthropatia[AII Fields] OR osteoarthropatias [AII Fields] OR osteoarthropatic [AII Fields] OR osteoarthropatie[AII Fields] OR osteoarthropaties[AII Fields] OR osteoarthrophy [All Fields] OR osteoarthrophytes [AII Fields] OR osteoarthroplasty [AII Fields] OR osteoarthros [AII Fields] OR osteoarthrose[AII Fields] OR osteoarthrosed[AII Fields] OR osteoarthroseentwicklung[All Fields] OR osteoarthrosen [AII Fields] OR osteoarthroser[AII Fields] OR osteoarthroses [AII Fields] OR osteoarthrosic [AII Fields] OR osteoarthrosique[AII Fields] OR osteoarthrosis [AII Fields] OR osteoarthrosis' [AII Fields] OR osteoarthrosis,[All Fields] OR

osteoarthrosisok [All Fields] OR osteoarthrositis[AII Fields] OR osteoarthrotendinous [All Fields] OR osteoarthrotenocutaneous[All Fields] OR osteoarthrotic [AII Fields] OR osteoarthrotically[AII Fields] OR osteoarthrotics [AII Fields] OR osteoarthrotis [AII Fields] OR osteoarthrotischen[AII Fields] OR osteoarthrotischer[AII Fields] OR osteoarthrotomy[All Fields]) OR (osteo arthrodesis[AII Fields] OR osteo
arthrodysplasia[All Fields] OR osteo arthropathia[All Fields] OR osteo arthropathic[All Fields] OR osteo arthropathie[AII Fields] OR osteo arthropathies[AII Fields]OR osteo arthropathique[AII Fields] OR osteo arthropathy[AII Fields] OR osteo arthropie[All Fields] OR osteo arthroplastic[AII Fields] OR osteo arthrose[All Fields] OR osteo arthroses[All Fields] OR osteo arthrosic[All Fields] OR osteo arthrosis[All Fields] OR osteo arthrotic[All Fields]) OR (osteoarthro[All Fields] OR osteoarthroapthy[All Fields] OR osteoarthrocutaneous[AII Fields] OR osteoarthrodermopathic[All Fields] OR osteoarthrodesis [AII Fields] OR osteoarthrodysplasia[AII Fields] OR osteoarthrography[AII Fields] OR osteoarthrologiai[AII Fields] OR osteoarthrological[AII Fields] OR osteoarthrology[AII Fields] OR osteoarthromuscular[AII Fields] OR osteoarthromusculovascular[All Fields] OR osteoarthromyalgias[AII Fields] OR osteoarthromyopathies[AII Fields] OR osteoarthroonychodysplasie[All Fields] OR osteoarthropathe[AII Fields] OR osteoarthropathi[AII Fields] OR osteoarthropathi'aja[All Fields] OR osteoarthropathia[All Fields] OR osteoarthropathiaja[AII Fields] OR osteoarthropathic [AII Fields] OR osteoarthropathie[AII Fields] OR osteoarthropathien [AII Fields] OR osteoarthropathies [AII Fields] OR osteoarthropathique[AII Fields] OR osteoarthropathy[AII Fields] OR osteoarthropathy1[AII Fields] OR osteoarthropatia[AII Fields] OR osteoarthropatias [AII Fields] OR osteoarthropatic [AII Fields] OR osteoarthropatie [AII Fields] OR osteoarthropaties[AII Fields] OR osteoarthrophy[AII Fields] OR osteoarthrophytes[AII Fields] OR osteoarthroplasty[AII Fields] OR osteoarthros [AII Fields] OR osteoarthrose[AII Fields] OR osteoarthrosed[AII Fields] OR osteoarthroseentwicklung[All Fields] OR osteoarthrosen[AII Fields] OR osteoarthroser[AII Fields] OR osteoarthroses[AII Fields] OR 
osteoarthrosic[AII Fields] OR osteoarthrosique [AII Fields] ORosteoarthrosis [AII Fields] OR osteoarthrosis' [AII Fields] OR osteoarthrosis, [AII Fields] OR osteoarthrosisok [All Fields] OR osteoarthrositis[AII Fields] OR osteoarthrotendinous[AII Fields] OR osteoarthrotenocutaneous[All Fields] OR osteoarthrotic [AII Fields] OR osteoarthrotically[AII Fields] OR osteoarthrotics [AII Fields] OR osteoarthrotis [AII Fields] OR osteoarthrotischen[AII Fields] OR osteoarthrotischer[AII Fields] OR osteoarthrotomy[AII Fields]) OR (gonarthritic[AII Fields] OR gonarthritiden[AII Fields] OR gonarthritides[All Fields] OR gonarthritis[All Fields]) OR (gonarthrocace[All Fields] OR gonarthrographe[AII Fields] OR gonarthroiis [AII Fields] OR gonarthromeningitis[All Fields] OR gonarthromuscular[AII Fields] OR gonarthromuskulares[AII Fields] OR gonarthropathies [AII Fields] OR gonarthropathy [AII Fields] OR gonarthroplasty [AII Fields] OR gonarthros [AII Fields] OR gonarthroscopy [AII Fields] OR gonarthrose[All Fields] OR

gonarthrosebehandlung[All Fields] OR gonarthrosen [AII Fie Ids ] OR gonarthrosepatienten[All Fields] OR gonarthroseprogedienz[All Fields] OR gonarthroses [A II FieIds] OR gonarthrosetherapie[All Fields] OR gonarthrosic [AII Fields ] OR gonarthrosique [AII Fields] OR gonarthrosiques [AII Fields] OR gonarthrosis [AII Fields] OR gonarthrosis'[All Fields] OR gonarthrotic[All Fields] OR gonarthroze[All Fields]) OR (coxarthritic[All Fields] OR coxarthritis[All Fields] OR coxarthritis"[All Fields]) OR (coxarthro[All Fields] OR coxarthrodesis[All Fields] OR coxarthrogenic[All Fields] OR coxarthrography [AII Fields] OR coxarthropathies [AIIFields] OR coxarthropathy [AII Fields] OR coxarthroplasty [AII Fields] OR coxarthros[All Fields] OR coxarthrose[All Fields] OR coxarthrosebehandlung[AII Fields] OR coxarthrosebehandlungen[All
Fields] OR coxarthrosefragen[All Fields] OR coxarthrosen [A II Fields] OR coxarthroseproblem[AII Fields] OR coxarthroses [AII FieIds] OR coxarthrosezeichen[AII Fields] OR coxarthrosic [AII Fields] OR coxarthrosique [AII Fields] OR coxarthrosiques [AII Fields] OR coxarthrosis[All Fields] OR coxarthrossi[All Fields] OR coxarthrotic[All Fields] OR coxarthroxis[All Fields] OR coxarthroz[All Fields]) OR (arthros[AII Fields] OR arthrosa[All Fields] OR arthrosas[All Fields] OR arthrosaura[All Fields] OR arthrosc[All Fields] OR arthroscan[All Fields] OR arthroscanner[AII Fields] OR arthroscanners[AII Fields] OR arthroscanning [AII Fields] OR arthroscanography[AII Fields] OR arthroscans [AII Fields] OR arthroscentesis [AII Fields] OR arthroscintigram [AII Fields] OR arthroscintigraphic[AII Fields] OR arthroscintigraphy[AII Fields] OR arthroscintography[AII Fields] OR arthroscipique [AII Fields] OR arthrosclerose[AII Fields] OR arthrosclerosis [AII Fields] OR arthrosclerotic[All Fields] OR arthrosco[All Fields] OR arthroscop[All Fields] OR arthroscopal[All Fields] OR arthroscope[All Fields] OR arthroscope's[All Fields] OR arthroscoped [A I I Fie Ids ] OR arthroscopes[All Fields] OR arthroscopi[All Fields] OR arthroscopia[All Fields] OR arthroscopially[AII Fields] OR arthroscopiaval[AII Fields] OR arthroscopic[AII Fields] OR arthroscopic'[AII Fields] OR arthroscopicacromioplasty[AII Fields] ORarthroscopical[AII Fields] OR arthroscopically [AII Fields] OR arthroscopicassisted[All Fields] OR arthroscopicIIy[AII Fields] OR arthroscopicmeniscectomy[All Fields] OR arthroscopico[AII Fields] OR arthroscopics [AII Fields] OR arthroscopie[AII Fields] OR arthroscopied[AII Fields] OR arthroscopies[AII Fields] OR arthroscopilally[All Fields] OR arthroscopique[AII Fields] OR arthroscopiques [AII Fields] OR arthroscopis [AII Fields] OR 
arthroscopist [AII Fields] OR arthroscopist's [AII Fields] OR arthroscopists [AII Fields] OR arthroscopists'[AII Fields] OR arthroscopoic[AII Fields] OR arthroscopos[All Fields] OR arthroscopy[All Fields] OR arthroscopy'[All Fields] OR arthroscopy's [AII Fields] OR arthroscopy1[AII Fields] OR arthroscopy 13 [AII Fields] OR arthroscopy2001[AII Fields] OR arthroscopya [AII Fields] OR arthroscopyando[AII Fields] OR arthroscopyassisted[AII Fields] OR arthroscopytechniques[All Fields] OR arthroscpec[All Fields] OR arthroscpic[All Fields] OR arthroscpoe[All Fields] OR arthroscpoic[All Fields] ORarthrose[All Fields] OR arthrose1[AII Fields] OR arthrose 1,2 [AII Fields] OR arthroseacetabula[AII Fields] OR arthroseassoziierte[AII Fields] OR arthroseauslosende[AII Fields] OR arthroseauslosung[All Fields] OR

arthrosebedingte [AII Fields] OR arthrosebehandeling[AII Fields] OR arthrosebehandlung[AII Fields] OR arthrosebeschwerden[All Fields] OR arthrosediagnostik[AII Fields] OR arthroseentstehung[AII Fields] OR arthroseentwicklung[AII Fields] OR arthroseforschung[AII Fields] OR arthroseforschungsverbundes[All Fields] OR arthrosegefahrdung[All Fields] OR arthrosegelenks [AII Fields] OR arthrosegrads [AII Fields] OR arthrosehaufigkeit[AII Fields] OR arthrosehilfe[AII Fields] OR arthroseindex[AII Fields] OR arthroseinduktion [All Fields] OR arthroseknie [AII Fields] OR arthrosekniegelenke[AII Fields] OR arthroseknorpeI[AII Fields] OR arthrosekranken[AII Fields] OR arthrosekrankheit[AII Fields] OR arthrosemerkmalen[AII Fields] OR arthrosemodell[All Fields] OR arthrosen[All Fields] OR arthrosenbehandlung[All Fields] OR arthrosenbildung[All Fields] OR arthrosendiagnostik[AII Fields] OR arthrosenepidemiologie[All Fields] OR arthrosenex [AII Fields] OR arthrosenpathogenese[All Fields] OR arthrosentherapie[AII Fields] OR
arthroseopy[All Fields] OR arthroseos[All Fields] ORarthrosepatienten[All Fields] OR arthrosepatienter [AII Fields] OR arthrosepatientin[AII Fields] OR arthroseproblem [AII Fields] OR arthroseprogression[AII Fields] OR arthroseprophylaktischen[All Fields] OR arthroseprophylaxe[AII Fields] OR arthroseprozess [AII Fields] OR arthroseptatienten[AII Fields] OR arthroser[All Fields] OR arthroseries[AII Fields] OR arthroserisiko[All Fields] OR arthroses [AII Fields] OR arthroseschmerz[AII Fields] OR arthroseschmerzen[AII Fields] OR arthroseschmerzes[AII Fields] OR arthrosetherapie[AII Fields] OR arthrosetten[AII Fields] OR arthroseursache[AII Fields] OR arthrosew[AII Fields] OR arthrosezeichen[All Fields] OR arthrosi[All Fields] OR arthrosia[All Fields] OR arthrosic[All Fields] OR arthrosico[All Fields] OR arthrosics[All Fields] OR arthroside[All Fields] OR arthrosides[All Fields] OR arthrosies[All Fields] OR arthrosim[All Fields] OR arthrosinovitis[All Fields] OR arthrosique[All Fields] OR arthrosiques[All Fields] OR arthrosis[All Fields] OR arthrosis'[All Fields] OR arthrosis a und [AII Fields] OR arthrosisban[All Fields] OR arthrosisra[All Fields] OR arthrositic[All Fields] OR arthrositis[All Fields] OR arthroskipische[All Fields] OR arthroskop[AIl Fields] OR arthroskope [AII Fields] OR arthroskopias [AII Fields] OR arthroskopiche[AII Fields] OR arthroskopie[AII Fields] OR arthroskopiebefund[AII Fields] OR arthroskopiebefunde[AII Fields] OR arthroskopien[AII Fields] OR arthroskopietagung[AII Fields] OR arthroskopisch [AII Fields] OR arthroskopische[AII Fields] OR arthroskopischem[AII Fields] OR arthroskopischen[AII Fields] OR arthroskopischer[AII Fields] OR arthroskopisches[AII Fields] OR arthroskopisher[All Fields] OR arthroso[AII Fields] OR arthrosoarthritis[All Fields] OR arthros oc opic [AII Fields] OR arthrosocopy[All Fields] OR arthrosocpy[All Fields] OR arthrosonografie[All Fields] OR 
arthrosonografische[All Fields] OR arthrosonogram [AII Fields] OR arthrosonographic[All Fields] OR arthrosonographical[AII Fields] OR arthrosonographically[All Fields] OR arthrosonographie[All Fields] OR arthrosonography[AII Fields] OR arthrosopic[AII FieIds] OR arthrosopically[AII Fields] OR arthrosopy[All Fields] OR arthrospec[All Fields] OR arthrosphaera[All

Fields] OR arthrosphaerae[All Fields] ORarthrosphaeridae[AII Fields] OR arthrosphores[All Fields] OR arthrospine[All Fields] OR arthrospira[All Fields] OR arthrospirai[AII Fields] OR arthrospiraplatensis[AII Fields] OR arthrosplasty[All Fields] OR arthrospora[All Fields] OR arthrospore[All Fields] OR arthrospores[All Fields] OR arthrosporic[All Fields] OR arthrosporiella[All Fields] OR arthrosporioides [AII Fields] OR arthrosporium [AII Fields] OR arthrosporogenesis [All Fields] OR arthrosporol[AII FieIds] OR arthrosporols [AII Fields] OR arthrosporone [AII Fields ] OR arthrosporous[All Fields] OR arthrosport[All Fields] OR arthrosporulating[All Fields] OR arthrosporulation [All Fields] OR arthrostasis[All Fields] OR arthrosteal[All Fields] OR arthrosteitis[All Fields] OR arthrostema [AII Fields ] OR arthrosteopedics [AII Fields] OR arthrostic[All Fields] OR arthrostigma[All Fields] OR arthrostoma[All Fields] OR arthrostomy[All Fields] OR arthrostrade[All Fields] OR arthrostress[All Fields] OR arthrostyla[AII Fields] OR arthrostylidiinae[AII Fields] OR arthrostylidioid[AII Fields] OR arthrosurface [AII Fields] OR arthrosurgery[All Fields] OR arthrosy[All Fields] OR arthrosynovial[All Fields] OR arthrosynoviaux [AII Fields] OR arthrosynovitic [All Fields] OR arthrosynovitis [AII Fields] OR arthrosyntheses [AII Fields] OR arthrosynthesis [AII Fields] OR arthroszintigrafie[AII Fields] OR arthroszkopiaja[AII Fields]) OR (arthrotammy[AII Fields] OR arthrotardigrada[AII Fields] OR arthrotardigrade [AII Fields] OR arthrotardigrades [AII Fields] OR arthrotec[All Fields] OR arthrotec's[AII Fields] OR arthrotek[All Fields] OR arthrotenodesis [AII Fields] OR arthrotenolyse [AII Fields] OR arthrotenolysis[All Fields] OR arthroteq[All Fields] OR arthrotera[All Fields] OR arthroteras[All Fields] ORarthroteres[All Fields] OR arthroteros[AII Fields] OR arthrotest[All Fields] OR arthrotetraose[All Fields] OR arthrothamnaceae[All Fields] OR arthrothamnus[All Fields] OR arthrotic[All Fields] OR arthrotical[All Fields] OR arthrotically[All Fields] OR arthroticum[All Fields] OR arthrotiker[All Fields] OR arthrotisch[All Fields] OR arthrotische[All Fields] OR arthrotischem[All Fields] OR arthrotischen [AII Fields] OR arthrotischer[AII Fields] OR arthrotmeterrel[AII Fields] OR arthrotome[All Fields] OR arthrotomein[All Fields] OR arthrotomi[AllFields] OR arthrotomia[All Fields] OR arthrotomic[All Fields] OR arthrotomie[All Fields] OR arthrotomies[All Fields] OR arthrotomii[All Fields] OR arthrotomised[All Fields] OR arthrotomized[AII Fields] OR arthrotomodensitometrique[All Fields] OR arthrotomodensitometry[All Fields] OR arthrotomogram [AII Fields] OR arthrotomogrames[AII Fields] OR arthrotomograms [AII Fields] OR arthrotomographic[AII Fields] OR arthrotomographically[All Fields] OR arthrotomographie[AII Fields] OR arthrotomographies[AII Fields] OR arthrotomographique[AII Fields] OR arthrotomographs [AII Fields] OR arthrotomography[AII Fields] OR arthrotomolgraphy[AII Fields] OR arthrotomty[All Fields] OR arthrotomy[All Fields] OR arthrotoxicity[All Fields] OR arthrotriaosylceramide[All Fields] OR arthrotrichus [AII Fields] OR arthrotripsometer[AII Fields] OR arthrotrophische[AII Fields] OR arthrotropic [AII Fields] OR arthrotropique [AII Fields] OR arthrotropism[All Fields] OR arthrotropy[All Fields] OR arthrotunneler[All Fields] OR arthrotus[All Fields])OR ("degenerative"[All Fields] AND "joint"[All Fields]

AND "disease"[AII Fields]) OR "degenerative joint disease"[All Fields]) 
AND ( ("hydroxymethylglutaryl-coa reductase inhibitors"[Pharmacological Action] OR "hydroxymethylglutaryl-coa reductase inhibitors"[MeSH Terms] OR ("hydroxymethylglutaryl-coa"[All Fields] AND "reductase"[AII Fields]AND "inhibitors" [AII Fields]) OR "hydroxymethylglutaryl-coa reductase inhibitors"[All Fields] OR "statin"[AII Fields]) OR ("hydroxymethylglutaryl-coa reductase inhibitors"[Pharmacological Action] OR "hydroxymethylglutaryl-coa reductase inhibitors"[MeSH Terms] OR ("hydroxymethylglutaryl-coa"[All Fields] AND "reductase"[All Fields] AND "inhibitors"[AII Fields]) OR "hydroxymethylglutaryl-coa reductase inhibitors"[All Fields] OR "statin"[AII Fields]) OR *statin OR("atorvastatin"[MeSH Terms] OR "atorvastatin"[All Fields]) OR ("cerivastatin"[Supplementary Concept] OR "cerivastatin "[AII Fields]) OR ("crilvastatin"[Supplementary Concept] OR "crilvastatin" [AII Fields]) OR ("Iovastatin"[MeSH Terms] OR "I ovastatin "[AII FieIds]) OR ("mevastatin"[Supplementary Concept] OR " mevastatin" [ A II Fie Ids ]) OR ("pitavastatin"[Supplementary Concept] OR "pitavastatin"[AII Fields]) OR ("pravastatin"[MeSH Terms] OR "pravastatin"[All Fields]) OR ("rosuvastatin c a I cium" [ M e S H Terms ] OR ("rosuvastatin"[AII Fields] AND "calcium"[All Fields]) OR "rosuvastatin calcium"[All Fields] OR "rosuvastatin"[All Fields]) OR ("simvastatin"[MeSH Terms] OR "simvastatin"[All Fields])).

Cochrane library - (In the topic of orthopedics \& trauma, endocrine \&metabolic, and rheumatology)

(Statin OR *statinOR Atorvastatin OR cerivastatin OR crilvastatin OR Lovastatin OR mevastatin OR pitavastatin OR Pravastatin OR Rosuvastatin OR Simvastatin) AND (Osteoarthrit* OR osteoarthrit* OR osteoarthro* OR osteo-arthro* OR "degenerative arthritis"OR osteoarthritis)

Embase - ('statin'/exp OR statinOR 'atorvastatin'/exp OR atorvastatinOR

'cerivastatin'/exp OR cerivastatinOR

'crilvastatin'/exp OR crilvastatinOR

'lovastatin'/exp OR lovastatinOR 'mevastatin'/exp OR mevastatinOR 'pitavastatin'/exp OR pitavastatinOR 'pravastatin'/exp OR pravastatinOR 'rosuvastatin'/exp OR rosuvastatinOR 'simvastatin'/exp OR simvastatin) AND ('osteoarthritis'/exp OR osteoarthritis)

Web of Science - ((Statin OR *statin OR Atorvastatin OR cerivastatin OR crilvastatin OR Lovastatin OR mevastatin OR pitavastatin OR Pravastatin OR Rosuvastatin OR Simvastatin) AND (Osteoarthrit* OR osteo-arthrit* OR osteoarthro* OR osteo-arthro* OR "degenerative arthritis" OR osteoarthritis)) Scopus - ((Statin OR *statin OR Atorvastatin OR cerivastatin OR crilvastatin OR Lovastatin OR mevastatin OR pitavastatin OR Pravastatin OR Rosuvastatin OR Simvastatin) AND (Osteoarthrit* OR osteoarthro* OR "degenerative arthritis" OR osteoarthritis)). 